\title{
Short Hydration in Chemotherapy with Cisplatin plus S-1 for Advanced or Recurrent Gastric Cancer: A Retrospective Study
}

\author{
Akihito Tsuji 1,2,3, Yuji Negoro3, Yoshihiro Okita ${ }^{1,2}$, Masahito Kotaka ${ }^{4}$, Takamasa Nishiuchi², \\ Takeshi Kotake', Hironaga Satake ${ }^{1}$, Yukimasa Hatachi ${ }^{1}$ \\ ${ }^{1}$ Department of Medical Oncology, Kobe City Medical Center General Hospital, Hyogo, Japan \\ ${ }^{2}$ Department of Clinical Oncology, Faculty of Medicine, Kagawa University, Kagawa, Japan \\ ${ }^{3}$ Department of Medical Oncology, Kochi Health Sciences Center, Kochi, Japan \\ ${ }^{4}$ Gastrointestinal Cancer Center, Sano Hospital, Hyogo, Japan \\ Email: a-tsuji@r4.dion.ne.jp
}

Received 4 August 2015; accepted 21 December 2015; published 25 December 2015

Copyright (C) 2015 by authors and Scientific Research Publishing Inc.

This work is licensed under the Creative Commons Attribution International License (CC BY). http://creativecommons.org/licenses/by/4.0/

(c) (i) Open Access

\section{Abstract}

Background: Despite there are a few reports that assessed the S-1 + CDDP regimen with short hydration regimen for unresectable or metastatic gastric cancer, there is no consensus on the best regimen for short hydration. The aim of study was to evaluate the safety and the efficacy of S-1 plus cisplatin doublet chemotherapy with short hydration. Methods: S-1 was administered orally (p.o.) twice daily for the first 3 weeks of a 5-week cycle. Dose of S-1 administered was calculated according to the body surface area. CDDP was given as an intravenous (i.v.) infusion of $60 \mathrm{mg} / \mathrm{m}^{2}$ on day 8 of each cycle. Patients received the total of $1900 \mathrm{ml}$ infusion containing $1000 \mathrm{ml}$ of acetate Ringer's solution as pre- and post-hydraion. $300 \mathrm{ml}$ of $20 \%$ mannitol was administered as a diuretic. Results: 35 patients with unresectable or recurrent gastric cancer were enrolled. The reasons for termination of S-1 + CDDP were as follows: 21 (63.6\%) by progressive disease; 12 (31.4\%) by toxicity. Even though 12 of 35 patients (34.2\%) were discontinued S-1 + CDDP chemotherapy, only one patient was discontinued by Grade 2 of increased creatinine. TTF (time to progression) was 174 days ( 3 - 586 days), and the median of the total number of treatment cycles of S-1 + CDDP was 3.31. Median overall survival, as secondary endpoint, was 518 days. Conclusions: Our study suggested that the short hydration regimen is as safe and efficient as the continuous hydration regimen.

\section{Keywords}

Short Hydration, Cisplatin Plus S-1, Gastric Cancer 


\section{Introduction}

Gastric cancer is the most common form of cancer and the second leading cause of cancer death in Japan. Gastric cancer is a major disease not only in east Asian countries, but also in the world [1]. Since JCOG (Japan Clinically Oncology Group) 9912 trial revealed that S-1 monotherapy showed non-inferiority for the 5-FU continuous infusion regimen, S-1 monotherapy had been considered the standard first line regimen for unresectable or recurrent gastric cancer in Japan [2]. Since this trial, randomised controlled trials of various treatment regimens containing cisplatin (CDDP) have produced disappointing findings in patients with advanced gastric cancer. However, SPIRITS trial showed that S-1 + CDDP regimen was better survival compared to S-1 monotherapy; S-1 + CDDP has been regarded as the standard first line systemic therapy for unresectable or recurrent gastric cancer in Japan [3]. In turn, CDDP containing regimens, for example ECF (epirubicin, cisplatin, fluorouracil), DCF (docetaxel, cisplatin, fluorouracil), are also regarded as the standard first line regimen for unresectable or recurrent gastric cancer in US and Europe [4] (National Comprehensive Cancer Network (NCCN) Guidelines Version 2.2015 Gastric Cancer).

Mor V. et al. reported that outpatient hospital care of medical oncology is clinically equivalent to inpatient care, which causes no negative psychosocial effects, and costs less than inpatient care. Moreover, they reported that most of patients hoped to receive outpatient chemotherapy if possible [5]. To avoid the nephrotoxicity, previous cisplatin containing regimens needs the 24-h/hydration. Because of this weak point, cisplatin containing regimen has not been applied to outpatient chemotherapy. Although it has been reported that short hydration chemotherapy containing cisplatin was safe and efficient in lung cancer, there were a few reports in gastric cancer. On the other hand, there is no consensus on the best regimen for hydration. In this study, we assessed the S-1 + CDDP regimen with short hydration based on the NCCN Guidelines Template for patients with unresectable metastatic gastric cancer.

The aim of study was to evaluate the safety and the efficacy of S-1 plus CDDP doublet chemotherapy with short hydration.

\section{Patients and Treatment}

\subsection{Patients}

Between March 2010 and September 2012, we retrospectively evaluated 35 patients with unresectable or recurrent gastric cancer who received chemotherapy containing S-1 and cisplatin in our institutes. Patients with histologically proven metastatic or recurrent gastric cancer were eligible for the study. Other patient's eligibility criteria were as follows: an Eastern Cooperative Oncology Group (ECOG) performance status of 0 - 1; neutrophil count $\geq 1200 / \mathrm{mm}^{3}$, hemoglobin level $\geq 8.0 \mathrm{~g} / \mathrm{dL}$, platelet count $\geq 75,000 / \mathrm{m}^{3}$. In terms of renal function, either a serum creatinine level of $\leq 1.5 \mathrm{mg} / \mathrm{dL}$ and an estimated creatinine clearance of $\geq 60 \mathrm{~mL} / \mathrm{min}$ were required.

\subsection{Treatments}

S-1 was administered orally (p.o.) twice daily for the first 3 weeks of a 5-week cycle. Dose of S-1 administered was calculated according to the body surface area; less than $1.25 \mathrm{~m}^{2}, 40 \mathrm{mg} ; 1.25$ - $1.5 \mathrm{~m}^{2}, 50 \mathrm{mg}$; and greater than $1.5 \mathrm{~m}^{2}, 60 \mathrm{mg}$. CDDP was given as an intravenous (i.v.) infusion of $60 \mathrm{mg} / \mathrm{m}^{2}$ on day 8 of each cycle. Patients were received the total of $1900 \mathrm{ml}$ infusion containing $1000 \mathrm{ml}$ of acetate Ringer's solution as pre- and post-hydraion. $300 \mathrm{ml}$ of $20 \%$ mannitol was administered as a diuretic. Palonosetron $0.75 \mathrm{mg}$ i.v., dexamethasone $9.9 \mathrm{mg}$ i.v. and aprepitant $125 \mathrm{mg}$ p.o. were administered 1 hour before infusional chemotherapy (Figure 1). Aprepitant $80 \mathrm{mg}$ p.o. and dexamethasone $4 \mathrm{mg}$ p.o. were administered on days 2 and 3 . This treatment schedule was repeated every 5 weeks according to SPIRITS trial [3].

We investigated patients who altered regimen to short hydration from conventional hydration. When physician confirmed the safety of CDDP short hydration in conference, patients were administered inpatient chemotherapy to outpatient chemotherapy.

\subsection{Statistical Analysis}

The primary endpoint was safety. Secondary endpoints were: overall survival (OS) and time to treatment failure (TTF). Treatment administration was regulated by evaluation of blood cell count before the start of the treatment 
cycle. Every four to six weeks, lesions were evaluated and measured mainly on computed tomography (CT). Response was defined according to the Response Evaluation Criteria in Solid Tumours (RECIST) version 1.1. Adverse events were graded according to the National Cancer Institute Common Terminology Criteria for Adverse Events (CTCAE; version 4.0). We used Kaplan-Meier method and calculate survival curves, and log-rank test to make treatment comparisons. Statistical analysis was performed using IBM SPSS statistics version 22.0 (SPSS Inc., Chicago, IL).

This trial was conducted in accordance with the World Medical Association Declaration of Helsinki and Japanese Good Clinical Practice guidelines.

\section{Results}

\subsection{Patient Characteristics}

A total of 35 patients were enrolled. Patients characteristics were shown as follows: male/female 24/11; median age (range) 62.1 (33 - 79); Histological type intestinal/diffuse/AFP secreting adenocarcinoma/squamous cell carcinoma $15(42.8 \%) / 17$ (48.6\%)/1 (2.9\%)/2 (5.7\%) (Table 1). 32 patients were administered chemotherapy as inpatient chemotherapy, remaining 3 patients were induced chemotherapy as outpatient chemotherapy. 7 patients out of 35 were altered treatment regimen to short hydration from long hydration. All patients were induced chemotherapy with short hydration since March 2010.

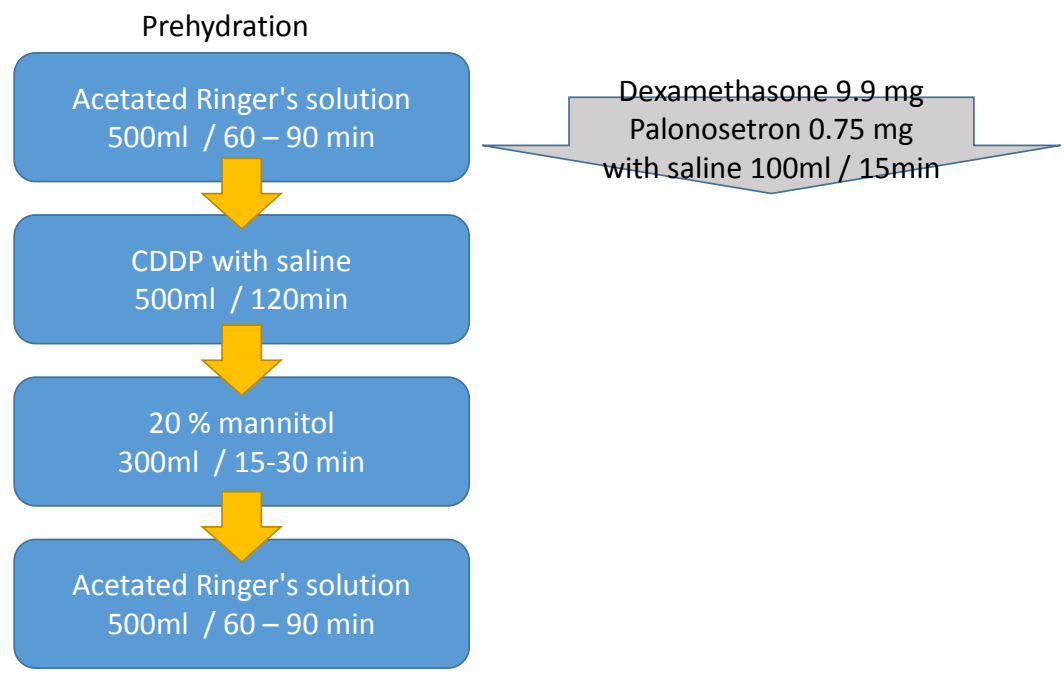

Total of $1900 \mathrm{ml}$ infusion with orally hydration per day

Figure 1. CDDP administration.

Table 1. Patient characteristics.

\begin{tabular}{lc}
\hline $\begin{array}{l}\text { Age } \\
\text { Gender }\end{array}$ & $62.1(33-79)$ \\
$\quad$ Male/Female & $24 / 11$ \\
Histological type & \\
Intestinal type & $15(42.8 \%)$ \\
Diffuse type & $17(48.6 \%)$ \\
AFP secreting adenocarcinoma & $1(2.9 \%)$ \\
Squamous cell carcinoma & $2(5.7 \%)$ \\
\hline
\end{tabular}




\subsection{Toxicity}

Two patients were continuing S-1 + CDDP treatment at the end of observation period. In remaining 33 patients, the reasons for termination of S-1 + CDDP were as follows: 20 (60.6\%) by progressive disease (PD); 10 (30.3\%) by toxicity. Toxicity was consists of five anorexia, one nausea, one diarrhea, one neutropenia, one Alanine aminotransferase increased, and one creatinine increased (Table 2(a), Table 2(b)). Even though 10 of 35 patients (28.6\%) were discontinued S-1 + CDDP chemotherapy by toxicity, only one patient were discontinued by Grade 2 of increased creatinine. Maximum creatinine level of the patient was $1.48 \mathrm{mg} / \mathrm{dL}$ on day 8 .

When we compared dose intensity of S-1 + CDDP between the reason of discontinuation by toxicity and progressive disease, the results were shown in Table 3. The mean age of toxicity group was 64.9 (47 - 74), and that of PD group was 60.3 (33 - 79). The mean dose of S-1 was $110.0 \mathrm{mg}$ in toxicity group and $111.4 \mathrm{mg}$ in PD groups. The mean dose of CDDP was $88.3 \mathrm{mg}$ in toxicity group and $92.7 \mathrm{mg}$ in PD group. Even if patients discontinued chemotherapy by toxicity, 5 of 10 patients $(50.0 \%)$ were able to be administered S-1 monotherapy. TTF including S-1 monotherapy followed by S-1 + CDDP discontinuation was slightly longer than only S-1 + CDDP therapy (204 days versus 174 days, Figure 2). In summary, we did not observe a significant difference between two groups.

Table 2. (a) Treatment interruption; (b) Distribution of reasons for treatment discontinuation.

(a)

\begin{tabular}{lc}
\hline & $\mathrm{n}=33$ \\
\hline Progressive disease & $20(60.6 \%)$ \\
Adverse events & $10(30.3 \%)$ \\
Anorexia & $5(15.2 \%)$ \\
Nausea & $1(3.0 \%)$ \\
Diarrhea & $1(3.0 \%)$ \\
Neutropenia & $1(3.0 \%)$ \\
Alanine aminotransferase increased & $1(3.0 \%)$ \\
Creatinine increased & $1(3.0 \%)$ \\
Cognitive disturbance & $2(6.1 \%)$ \\
Ileus & $1(3.0 \%)$
\end{tabular}

(b)

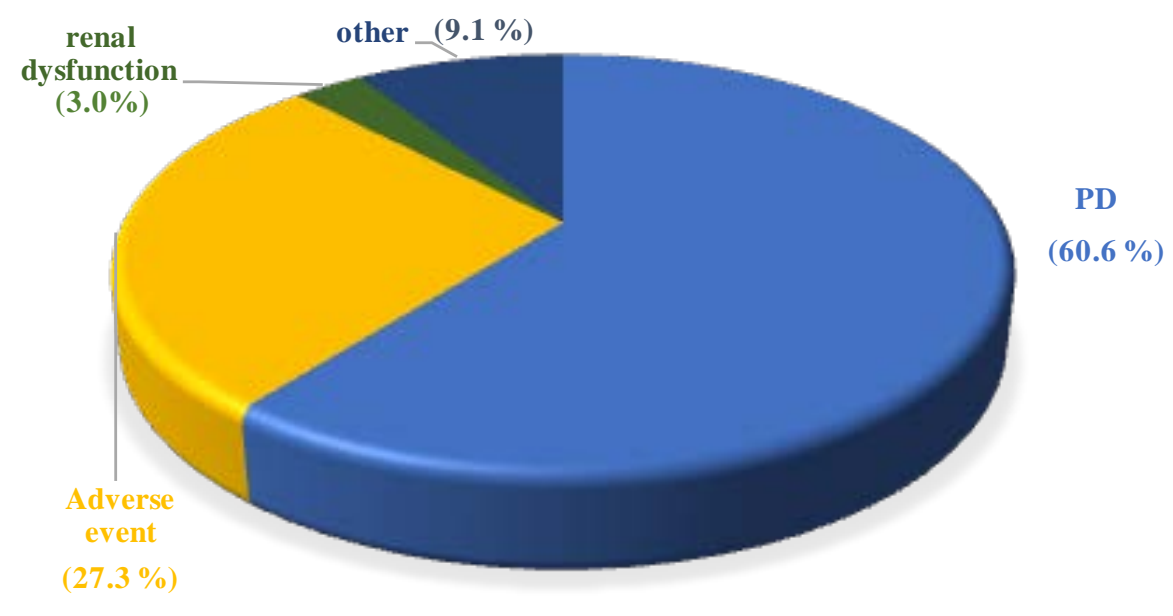

PD; progressive disease 
Table 3. Comparison of treatment discontinuation by toxicity and PD.

\begin{tabular}{ccc}
\hline & Toxicity & PD \\
\hline Age & $64.9(47-74)$ & $60.3(33-79)$ \\
Gender Male/Female & $8 / 2$ & $12 / 8$ \\
Dose of S-1 & $110.0 \mathrm{mg} / \mathrm{body}$ & $111.4 \mathrm{mg} / \mathrm{body}$ \\
Dose of CDDP & $88.3(76-103) \mathrm{mg} /$ body & $92.7(60-113) \mathrm{mg} / \mathrm{body}$ \\
$\begin{array}{c}\text { Secondary therapy } \\
\text { (including S-1 monotherapy) }\end{array}$ & $5(50.0 \%)$ & $3(15.0 \%)$ \\
\hline
\end{tabular}

PD: Progressive disease.

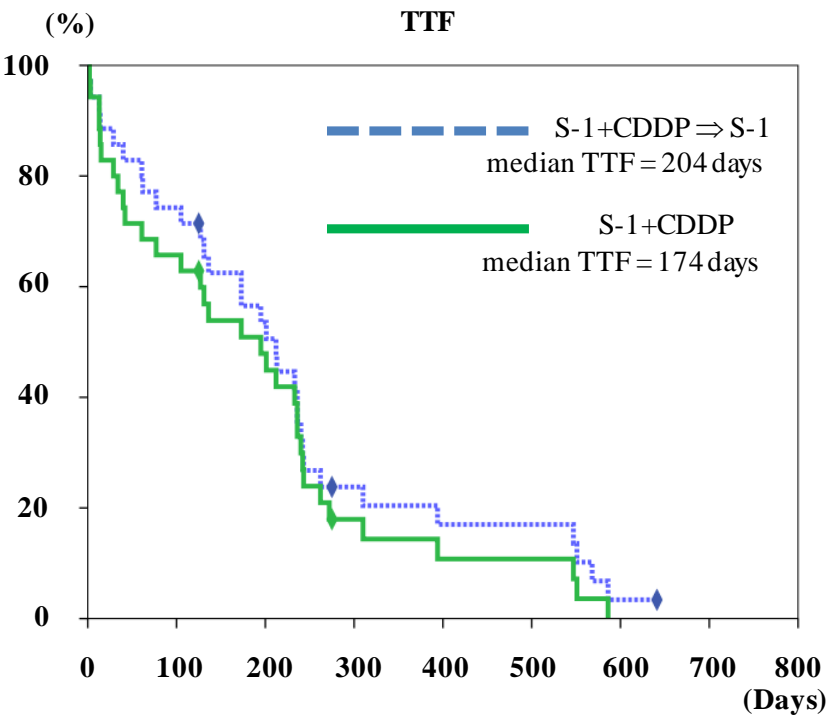

Figure 2. Kaplan-Meier curve of time to treatment failure (TTF) of S-1 monotherapy followed S-1 + CDDP discontinuation.

\subsection{Efficacy}

Duration of median follow up was 378 days (32 - 694 days). TTF as secondary endpoint was 174 days (3 - 586 days), and the median of the total number of treatment cycles of S-1 + CDDP were 3.31 (1 - 9) (Figure 3). Median overall survival, as secondary endpoint, was 518 days (32 - 683 days) (Figure 4).

\section{Discussion}

In the present study, we investigated the safety and efficacy of S-1 + CDDP short hydration for unresectable or recurrent gastric cancer.

Previous study validated short hydration containing CDDP ( $\geq 75 \mathrm{mg} / \mathrm{m}^{2} /$ cycle) in lung cancer. The study revealed the safety and efficacy antecedent to gastric cancer [6]. Another study compared 30 patients with gastric cancer, lung cancer, and the urothelial cancer who received outpatient chemotherapy containing CDDP ( $\geq 60$ $\mathrm{mg} / \mathrm{m}^{2} /$ cycle) with short hydration regimen to those who received hospital chemotherapy with continuous hydration. The study reported that there were no differences between two groups in creatinine level, and relative dose intensity in the short hydration group was higher than that in the continuous hydration group [7].

Okazaki et al. prospectively reported short hydration regimen of S-1 + CDDP for gastric cancer patients. Advanced gastric cancer patients received an S-1 + CDDP regimen, either as outpatient chemotherapy with oral hydration on day 9 to 10, or as inpatient chemotherapy with intravenous hydration on day 9 to 10, based on the results of an oral hydration test during day 1 to 7 of the first cycle. Patients were infused $1000 \mathrm{ml}$ of normal saline before premedication and $1000 \mathrm{ml}$ of normal saline after CDDP administration on day 8. Patients ingest 


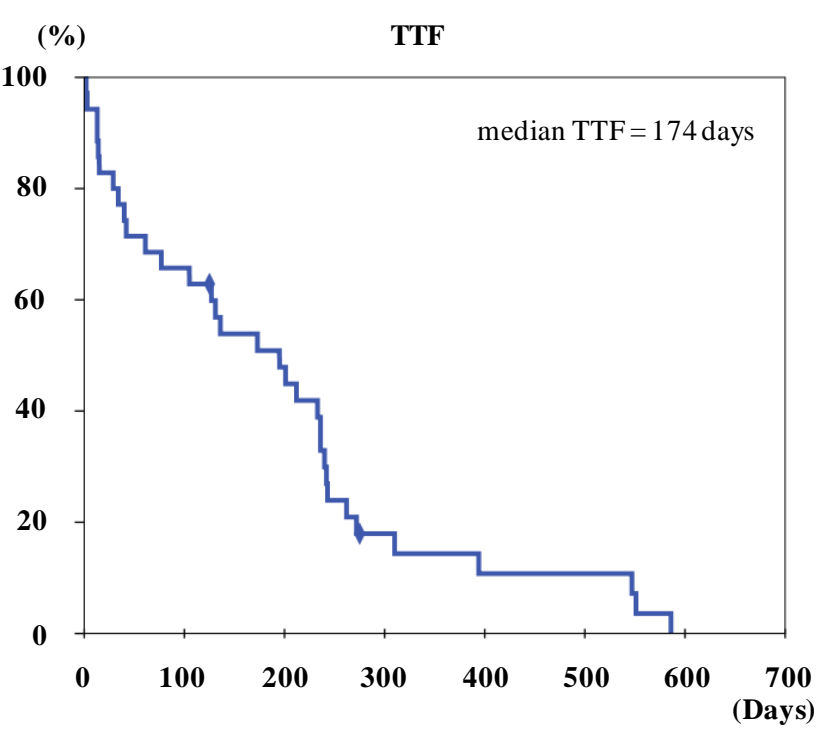

Figure 3. Kaplan-Meier curve of time to treatment failure (TTF).

(\%) OS

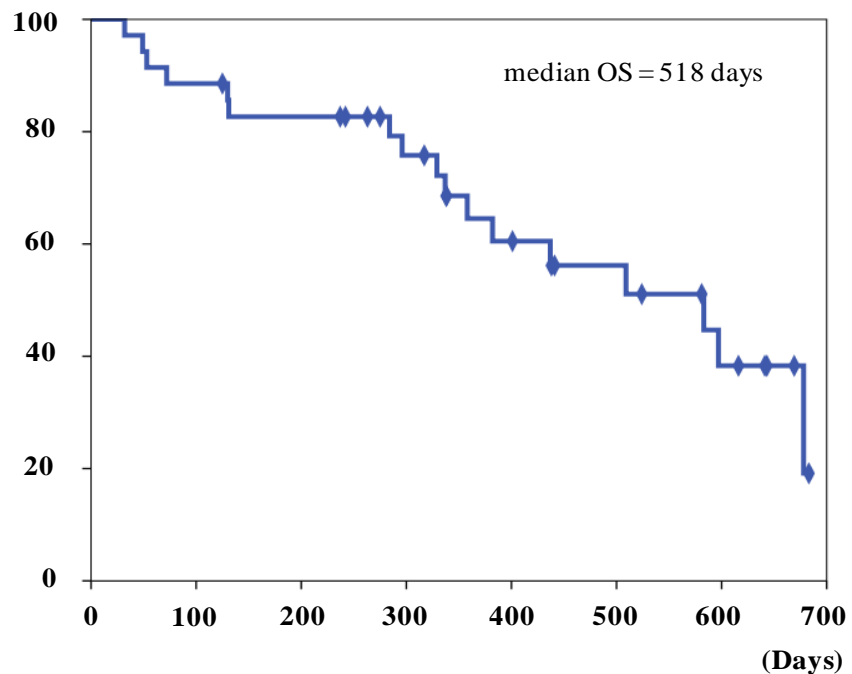

Figure 4. Kaplan-Meier curve of overall survival (OS).

orally $1500 \mathrm{ml}$ of water on day 9 and 10 [8]. Satake et al. who is the author in the present study reported that patients were infused $1000 \mathrm{ml}$ of $0.45 \% \mathrm{NaCl}$ with $\mathrm{Mg} 2.5 \mathrm{mEq}$ as prehydration and $500 \mathrm{ml}$ of $0.45 \% \mathrm{NaCl}$ with $\mathrm{Mg} 2.5 \mathrm{mEq}$ as posthydration with $1000 \mathrm{ml}$ of oral hydration. They administered total 2250 ml of hydration for four hours on day 8 [9].

Comparing our study and these previous studies, we did not identify the significant differentiation of patient characteristics, dose intensity, toxicity and survival. In this study, patients were administered hydration by only intravenous infusion without oral hydration. Despite less hydration, as a result, we considered that the nephrotoxicity of our regimen was similar as that of previous reports. Gastric cancer patients are relatively elder, and it is difficult especially for elder patients to apply the high dose oral hydration in outpatient chemotherapy. Therefore we considered that our hydration regimen was more feasible than previous two reports for outpatient chemotherapy.

In the SPIRITS trial, Grade 3 or 4 adverse events in the S-1 + CDDP arm were shown in Table 4 [3]. In this study, the reason for treatment discontinuation were almost less toxicity than SPIRITS study. Despite S-1 + CDDP regimen is highly emetogenic chemotherapy, we did not observe the treatment discontinuation by nausea 
Table 4. Comparison of Grade 3 - 4 adverse events between SPIRITS trial and our trial.

\begin{tabular}{ccc}
\hline & Our results $(\mathrm{n}=35)$ & SPIRITS trial $(\mathrm{n}=148)$ \\
\hline Anorexia & $6(17.1 \%)$ & $45(30 \%)$ \\
Nausea & $1(2.9 \%)$ & $17(11 \%)$ \\
Diarrhea & $1(2.9 \%)$ & $6(4 \%)$ \\
Neutropenia & $1(2.9 \%)$ & $59(40 \%)$ \\
Increased creatinine & $1(2.9 \%)$ & 0 \\
\hline
\end{tabular}

and vomiting in our study. This result was leaded according to the progression of anti-emetic therapy compared with SPIRITS era. Previous reports suggest that the triplet anti-emetic regimen comprising aprepitant, palonosetron, and dexamethasone was useful for cisplatin-based highly emetogenic chemotherapy [10] [11].

The present study has several limitations. Limitation was as follows: duration of follow up was short, small number of patients, retrospective study design.

Prospective phase 2 trial of Japan Southwest Research Support Organization Group (JSWOG)-G1 study to evaluate feasibility of S-1 + CDDP chemotherapy with short hydration is undergoing, there is further discussion expected in the future.

\section{Conclusion}

Our study suggested that the short hydration regimen was as safe and efficient as the continuous hydration regimen.

\section{References}

[1] Tanaka, M., Ma, E., Tanaka, H., Ioka, A., Nakahara, T. and Takahashi, H. (2012) Trends of Stomach Cancer Mortality in Eastern Asia in 1950-2004: Comparative Study of Japan, Hong Kong and Singapore Using Age, Period and Cohort Analysis. International Journal of Cancer, 130, 930-936. http://dx.doi.org/10.1002/ijc.26080

[2] Boku, N., Yamamoto, S., Fukuda, H., Shirao, K., Doi, T., Sawaki, A., Koizumi, W., Saito, H., Yamaguchi, K., Takiuchi, H., Nasu, J. and Ohtsu, A. (2009) Gastrointestinal Oncology Study Group of the Japan Clinical Oncology G: Fluorouracil versus Combination of Irinotecan plus Cisplatin versus S-1 in Metastatic Gastric Cancer: A Randomised Phase 3 Study. The Lancet Oncology, 10, 1063-1069. http://dx.doi.org/10.1016/S1470-2045(09)70259-1

[3] Koizumi, W., Narahara, H., Hara, T., Takagane, A., Akiya, T., Takagi, M., Miyashita, K., Nishizaki, T., Kobayashi, O., Takiyama, W., Toh, Y., Nagaie, T., Takagi, S., Yamamura, Y., Yanaoka, K., Orita, H. and Takeuchi, M. (2008) S-1 plus Cisplatin versus S-1 Alone for First-Line Treatment of Advanced Gastric Cancer (Spirits Trial): A Phase III Trial. The Lancet Oncology, 9, 215-221. http://dx.doi.org/10.1016/S1470-2045(08)70035-4

[4] Waddell, T., Verheij, M., Allum, W., Cunningham, D., Cervantes, A., Arnold, D., European Society for Medical Oncology (ESMO), European Society of Surgical Oncology (ESSO) and European Society of Radiotherapy and Oncology (ESTRO) (2013) Gastric Cancer: ESMO-ESSO-ESTRO Clinical Practice Guidelines for Diagnosis, Treatment and Follow-Up. Annals of Oncology, 24, 57-63. http://dx.doi.org/10.1093/annonc/mdt344

[5] Mor, V., Stalker, M.Z., Gralla, R., Scher, H.I., Cimma, C., Park, D., Flaherty, A.M., Kiss, M., Nelson, P., Laliberte, L., et al. (1988) Day Hospital as an Alternative to Inpatient Care for Cancer Patients: A Random Assignment Trial. Journal of Clinical Epidemiology, 41, 771-785. http://dx.doi.org/10.1016/0895-4356(88)90164-3

[6] Horinouchi, H., Kubota, K., Itani, H., Taniyama, T.K., Nakamichi, S., Wakui, H., Kanda, S., Nokihara, H., Yamamoto, N., Sekine, I. and Tamura, T. (2013) Short Hydration in Chemotherapy Containing Cisplatin $\left(>/=75 \mathrm{mg} / \mathrm{m}^{2}\right)$ for Patients with Lung Cancer: A Prospective Study. Japanese Journal of Clinical Oncology, 43, 1105-1109. http://dx.doi.org/10.1093/jjco/hyt122

[7] Ouchi, A., Asano, M., Aono, K., Watanabe, T. and Kato, T. (2014) Comparison of Short and Continuous Hydration Regimen in Chemotherapy Containing Intermediate- to High-Dose Cisplatin. Journal of Oncology, 2014, Article ID: 767652. http://dx.doi.org/10.1155/2014/767652

[8] Okazaki, S., Nakajima, T.E., Hashimoto, J., Yamamoto, S., Takahari, D., Kato, K., Hamaguchi, T., Yamada, Y., Shimada, Y. and Tamura, K. (2013) A Feasibility Study of Outpatient Chemotherapy with s-1 + Cisplatin in Patients with Advanced Gastric Cancer. Gastric Cancer, 16, 41-47. http://dx.doi.org/10.1007/s10120-012-0139-4

[9] Satake, H., Tsuji, A., Kotake, T. and Fujita, M. (2014) Feasibility of Outpatient Chemotherapy with S-1 and Cisplatin 
for Gastric Cancer. Journal of Cancer Therapy, 5, 759-765. http://dx.doi.org/10.4236/jct.2014.58084

[10] Kitazaki, T., Fukuda, Y., Fukahori, S., Oyanagi, K., Soda, H., Nakamura, Y. and Kohno, S. (2015) Usefulness of antiemetic Therapy with Aprepitant, Palonosetron, and Dexamethasone for Lung Cancer Patients on Cisplatin-Based or Carboplatin-Based Chemotherapy. Supportive Care in Cancer, 23, 185-190.

http://dx.doi.org/10.1007/s00520-014-2339-4

[11] Longo, F., Mansueto, G., Lapadula, V., Stumbo, L., Del Bene, G., Adua, D., De Filippis, L., Bonizzoni, E. and Quadrini, S. (2012) Combination of Aprepitant, Palonosetron and Dexamethasone as Antiemetic Prophylaxis in Lung Cancer Patients Receiving Multiple Cycles of Cisplatin-Based Chemotherapy. International Journal of Clinical Practice, 66, 753-757. http://dx.doi.org/10.1111/j.1742-1241.2012.02969.x 